\title{
On certain functional equations related to Jordan $*$-derivations in semiprime $*$-rings and standard operator algebras
}

\author{
MOHAMmad ASHRAF \\ Department of Mathematics \\ Aligarh Muslim University \\ Aligarh-202002 India \\ email: mashraf80@hotmail.com \\ and \\ Bilal Ahmad Wani \\ Department of Mathematics \\ Aligarh Muslim University \\ Aligarh-202002 India \\ email: bilalwanikmr@gmail.com
}

(Received: April 17, 2017, and in revised form October 27, 2017.)

\begin{abstract}
The purpose of this paper is to investigate identities with Jordan *-derivations in semiprime *-rings. Let $\mathcal{R}$ be a 2-torsion free semiprime $*$-ring. In this paper it has been shown that, if $\mathcal{R}$ admits an additive mapping $D: \mathcal{R} \rightarrow \mathcal{R}$ satisfying either $D(x y x)=D(x y) x^{*}+x y D(x)$ for all $x, y \in \mathcal{R}$, or $D(x y x)=D(x) y^{*} x^{*}+x D(y x)$ for all pairs $x, y \in \mathcal{R}$, then $D$ is a $*$-derivation. Moreover this result makes it possible to prove that if $\mathcal{R}$ satisfies $2 D\left(x^{n}\right)=D\left(x^{n-1}\right) x^{*}+x^{n-1} D(x)+D(x)\left(x^{*}\right)^{n-1}+x D\left(x^{n-1}\right)$ for all $x \in \mathcal{R}$ and some fixed integer $n \geq 2$, then $D$ is a Jordan $*$-derivation under some torsion restrictions. Finally, we apply these purely ring theoretic results to standard operator algebras $\mathcal{A}(\mathcal{H})$. In particular, we prove that if $\mathcal{H}$ be a real or complex Hilbert space, with $\operatorname{dim}(\mathcal{H})>1$, admitting a linear mapping $D: \mathcal{A}(\mathcal{H}) \rightarrow \mathcal{B}(\mathcal{H})$ (where $\mathcal{B}(\mathcal{H})$ stands for the bounded linear operators) such that
\end{abstract}

$$
2 D\left(A^{n}\right)=D\left(A^{n-1}\right) A^{*}+A^{n-1} D(A)+D(A)\left(A^{*}\right)^{n-1}+A D\left(A^{n-1}\right)
$$

for all $A \in \mathcal{A}(\mathcal{H})$. Then $D$ is of the form $D(A)=A B-B A^{*}$ for all $A \in \mathcal{A}(\mathcal{H})$ and some fixed $B \in \mathcal{B}(\mathcal{H})$, which means that $D$ is Jordan $*$-derivation.

Mathematics Subject Classification(2010). 16N60, 16W10, 46K15, 16W25.

Keywords: prime ring, semiprime ring, standard operator algebra, Jordan $*$ derivation, Jordan triple $*$-derivation.

\section{Introduction}

Throughout this paper $\mathcal{R}$ will denote an associative ring with center $Z(\mathcal{R})$. Recall that a ring $\mathcal{R}$ is said to be $n$-torsion free, where $n>1$ is an integer, if $n x=0$ implies $x=0$ for all $x \in \mathcal{R}$. For any $x, y \in \mathcal{R}$, the symbol $[x, y]$ will denote the commutator $x y-y x$. A ring $\mathcal{R}$ is said to be prime if for any $a, b \in \mathcal{R}, a \mathcal{R} b=\{0\}$ implies $a=0$ or $b=0$, and $\mathcal{R}$ is semiprime if for any $a \in \mathcal{R}, a \mathcal{R} a=\{0\}$ implies $a=0$. An additive mapping $x \mapsto x^{*}$ on a ring $\mathcal{R}$ is called involution in case $(x y)^{*}=y^{*} x^{*}$ and $\left(x^{*}\right)^{*}=x$ hold for all $x, y \in \mathcal{R}$. A ring equipped with an involution is called a ring with involution or $*$-ring (see

DOI: $10.1515 /$ puma-2015-0024 
[13]). An additive mapping $D: \mathcal{R} \rightarrow \mathcal{R}$ is said to be a derivation on $\mathcal{R}$ if $D(x y)=D(x) y+x D(y)$ for all $x, y \in \mathcal{R}$ and is called a Jordan derivation if $D\left(x^{2}\right)=D(x) x+x D(x)$ for all $x \in \mathcal{R}$. A derivation $D$ is inner if there exists $a \in \mathcal{R}$ such that $D(x)=[a, x]$ holds for all $x \in \mathcal{R}$. A Jordan triple derivation $D: \mathcal{R} \rightarrow \mathcal{R}$ is an additive mapping if $D(x y x)=D(x) y x+x D(y) x+x y D(x)$ for all $x, y \in \mathcal{R}$. It is clear that any derivation is a Jordan derivation. The converse is in general not true. A classical result of Herstein [11] asserts that every Jordan derivation on a prime ring of characteristic different from two is a derivation. A brief proof of Herstein's result can be found in [6]. If $\mathcal{R}$ is 2-torsion free, it can be easily proved that any Jordan derivation $D: \mathcal{R} \rightarrow \mathcal{R}$ is a Jordan triple derivation (see [12]). A famous result due to Brešar [5, Theorem 4.3], asserts that a Jordan triple derivation on a 2-torsion free semiprime ring is a derivation. Following the same line, a number of results have been obtained by several authors (see [2], [3], [4], [9], [18], [19], [22, [23]), where further references can be found.

Let $\mathcal{R}$ be a $*$-ring. An additive mapping $D: \mathcal{R} \rightarrow \mathcal{R}$ is said to be a $*$-derivation on $\mathcal{R}$ if $D(x y)=$ $D(x) y^{*}+x D(y)$ for all $x, y \in \mathcal{R}$ and is called a Jordan $*$-derivation if $D\left(x^{2}\right)=D(x) x^{*}+x D(x)$ holds for all $x \in \mathcal{R}$ Note that the mapping $x \mapsto x a-a x^{*}$, where $a$ is a fixed element in $\mathcal{R}$, is a Jordan *-derivation. Such a Jordan $*$-derivation is said to be inner. The study of Jordan $*$-derivations has been motivated by the problem of the representativity of quadratic forms by bilinear forms (for the results concerning this problem we refer the reader to [8], [15], and [16]). It turns out that the question, whether each quadratic form can be represented by some bilinear form, is connected with the question, whether every Jordan $*$-derivation is inner, as shown by Šemrl [15].

A Jordan triple $*$-derivation is an additive mapping $D: \mathcal{R} \rightarrow \mathcal{R}$ with the property $D(x y x)=$ $D(x) y^{*} x^{*}+x D(y) x^{*}+x y D(x)$ for all $x, y \in \mathcal{R}$. One might expect that any Jordan $*$-derivation on a 2 -torsion free semiprime $*$-ring is a $*$-derivation, but this is not the case. It is easy to prove that there are no nonzero $*$-derivations on noncommutative prime $*$-rings (see [7] for the details). Any Jordan *-derivation $D: \mathcal{R} \rightarrow \mathcal{R}$ on a 2 -torsion free $*$-ring $\mathcal{R}$ is a Jordan triple $*$-derivation. However, the converse of this statement is not true in general (see [1]). In [24], Vukman showed that the converse holds if $\mathcal{R}$ is 6 -torsion free semiprime $*$-ring. Recently, Fošner and Iliševič [10] proved that every Jordan triple $*$-derivation on a 2 -torsion free semiprime $*$-ring is a Jordan $*$-derivation. In view of these results we begin our investigation with additive mapping $D$ on a semiprime $*$-ring $\mathcal{R}$ which satisfies either of the identities $D(x y x)=D(x y) x^{*}+x y D(x)$ or $D(x y x)=D(x) y^{*} x^{*}+x D(y x)$ and show that $D$ is a *-derivation on $\mathcal{R}$. Further, it is shown that if the additive mapping $D$ satisfies either of the properties $D(x y x)=D(x y) x^{*}-x y D(x)$ or $D(x y x)=D(x) y^{*} x^{*}-x D(y x)$, then $D$ is a Jordan $*$-derivation. Finally, a result concerning the identity $2 D\left(x^{n}\right)=D\left(x^{n-1}\right) x^{*}+x^{n-1} D(x)+D(x)\left(x^{*}\right)^{n-1}+x D\left(x^{n-1}\right)$ has also been obtained.

\section{Results on semiprime $*$-ring}

We begin with the following results which are crucial for developing the proof of our main results.

Lemma 2.1 [22, Lemma 3] Let $\mathcal{R}$ be a semiprime ring and $f: \mathcal{R} \rightarrow \mathcal{R}$ be an additive mapping such that either $f(x) x=0$ or $x f(x)=0$ for all $x \in \mathcal{R}$. Then $f=0$.

Now we will prove the following main results. 
THEOREM 2.2 Let $\mathcal{R}$ be a 2-torsion free semiprime $*$-ring and $D: \mathcal{R} \rightarrow \mathcal{R}$ be an additive mapping such that either

$$
D(x y x)=D(x y) x^{*}+x y D(x), \text { for all } x, y \in \mathcal{R}
$$

or

$$
D(x y x)=D(x) y^{*} x^{*}+x D(y x), \text { for all } x, y \in \mathcal{R} .
$$

Then $D$ is a Jordan $*$-derivation, whence $D$ is a $*$-derivation. In fact, we can conclude that $D(\mathcal{R})$ generates a central ideal of $\mathcal{R}$.

Proof. We will restrict our attention on the relation $(1)$, the proof in case when $\mathcal{R}$ satisfies the relation (2) is similar and will therefore be omitted. Linearization of the relation (1) gives

$$
D(x y z+z y x)=D(x y) z^{*}+D(z y) x^{*}+x y D(z)+z y D(x),
$$

for all $x, y, z \in \mathcal{R}$. In particular for $z=x^{2}$, the above relation gives

$$
D\left(x y x^{2}+x^{2} y x\right)=D(x y) x^{*^{2}}+D\left(x^{2} y\right) x^{*}+x y D\left(x^{2}\right)+x^{2} y D(x),
$$

for all $x, y, \in \mathcal{R}$. Putting $x y+y x$ for $y$ in (1) and applying the relation (1), we obtain

$$
\begin{aligned}
D\left(x y x^{2}+x^{2} y x\right)= & D\left(x^{2} y\right) x^{*}+D(x y) x^{*^{2}}+x y D(x) x^{*} \\
& +x^{2} y D(x)+x y x D(x),
\end{aligned}
$$

for all $x, y \in \mathcal{R}$. By comparing (3) and (4), we have

$$
x y A(x)=0, \text { for all } x, y \in \mathcal{R},
$$

where $A(x)$ stands for $D\left(x^{2}\right)-D(x) x^{*}-x D(x)$. Right multiplication of 5 by $x$ and left multiplication by $A(x)$ gives,

$$
A(x) x y A(x) x=0, \text { for all } x, y \in \mathcal{R} .
$$

By the semiprimeness of $\mathcal{R}$, it follows that

$$
A(x) x=0 \text {, for all } x \in \mathcal{R} .
$$

The substitution of $A(x) y x$ for $y$ in the relation (5), gives $x A(x) y x A(x)=0$ for all pairs $x, y \in \mathcal{R}$. Hence, we obtain

$$
x A(x)=0, \text { for all } x \in \mathcal{R} .
$$

The linearization of the relation $(6)$ gives

$$
B(x, y) x+A(x) y+B(x, y) y+A(y) x=0
$$


for all pairs $x, y \in \mathcal{R}$, where $B(x, y)$ denotes $D(x y+y x)-D(x) y^{*}-x D(y)-D(y) x^{*}-y D(x)$. Putting in the above relation $-x$ for $x$ and comparing the relation so obtained with the above relation one obtains

$$
B(x, y) x+A(x) y=0 \text {, for all } x, y \in \mathcal{R} .
$$

In view of the relation $(7)$, right multiplication by $A(x)$ gives, $A(x) y A(x)=0$ for all pairs $x, y \in \mathcal{R}$. Hence it follows that $A(x)=0$ for all $x \in \mathcal{R}$. In other words, $D$ is a Jordan $*$-derivation. Hence it follows that $D$ is a Jordan triple $*$-derivation. Now, comparing the relation $D(x y x)=D(x) y^{*} x^{*}+$ $x D(y) x^{*}+x y D(x)$, for all $x, y \in \mathcal{R}$, with the relation (1), we get

$$
\left(D(x y)-D(x) y^{*}-x D(y)\right) x^{*}=0, \text { for all } x, y \in \mathcal{R} .
$$

For any fixed $y \in \mathcal{R}$, we have an additive mapping $x \mapsto D(x y)-D(x) y^{*}-x D(y)$ on $\mathcal{R}$. Thus from the above relation and by the consequence of Lemma 2.1, it follows that $D(x y)-D(x) y^{*}-x D(y)=0$, for all pairs $x, y \in \mathcal{R}$. In other words, $D$ is a $*$-derivation. Hence by [14, Theorem 3.1], $D(\mathcal{R}) \subseteq Z(\mathcal{R})$. This completes the proof.

For the sake of brevity, we omit the proof of the following statement.

TheOREm 2.3 Let $\mathcal{R}$ be a 2-torsion free semiprime $*$-ring. Suppose $D: \mathcal{R} \rightarrow \mathcal{R}$ is an additive mapping such that either

$$
D(x y x)=D(x y) x^{*}-x y D(x), \text { for all } x, y \in \mathcal{R}
$$

or

$$
D(x y x)=D(x) y^{*} x^{*}-x D(y x), \text { for all } x, y \in \mathcal{R} .
$$

Then $D$ is a Jordan $*$-derivation. If, in addition, $1 \in \mathcal{R}$, then $D=0$.

Disadvantage of Theorem 2.2 is that in identities (1) and (2) there is no symmetry. Therefore, Theorem 2.2 , together with the desire for symmetry leads to the following conjecture.

Conjecture 2.4 Let $\mathcal{R}$ be a 2 -torsion free semiprime $*$-ring and $D: \mathcal{R} \rightarrow \mathcal{R}$ be an additive mapping such that

$$
2 D(x y x)=D(x y) x^{*}+x y D(x)+D(x) y^{*} x^{*}+x D(y x),
$$

holds for all pairs $x, y \in \mathcal{R}$. Then $D$ is a Jordan $*$-derivation.

Note that in case a ring has the identity element, the proof of the above conjecture is immediate. The substitution $y=e$ in the relation (10), where $e$ stands for the identity element, gives that $D$ is a Jordan $*$-derivation.

The substitution of $y=x^{n-2}$ in the relation 10 gives

$$
2 D\left(x^{n}\right)=D\left(x^{n-1}\right) x^{*}+x^{n-1} D(x)+D(x)\left(x^{*}\right)^{n-1}+x D\left(x^{n-1}\right),
$$

which leads to the following conjecture. 
Conjecture 2.5 Let $\mathcal{R}$ be a semiprime $*$-ring with a suitable torsion restriction and $D: \mathcal{R} \rightarrow \mathcal{R}$ be an additive mapping such that

$$
2 D\left(x^{n}\right)=D\left(x^{n-1}\right) x^{*}+x^{n-1} D(x)+D(x)\left(x^{*}\right)^{n-1}+x D\left(x^{n-1}\right),
$$

holds for all $x \in \mathcal{R}$ and some fixed integer $n \geq 2$. Then $D$ is a Jordan $*$-derivation.

Now we prove the above conjecture in case a ring has the identity element.

THEOREM 2.6 Let $\mathcal{R}$ be a $2(n-1)$ !-torsion free semiprime $*$-ring with identity e and $D: \mathcal{R} \rightarrow \mathcal{R}$ be an additive mapping such that

$$
2 D\left(x^{n}\right)=D\left(x^{n-1}\right) x^{*}+x^{n-1} D(x)+D(x)\left(x^{*}\right)^{n-1}+x D\left(x^{n-1}\right),
$$

for all $x \in \mathcal{R}$ and some fixed integer $n \geq 2$. Then $D$ is a Jordan $*$-derivation.

Proof. We have the relation

$$
2 D\left(x^{n}\right)=D\left(x^{n-1}\right) x^{*}+x^{n-1} D(x)+D(x)\left(x^{*}\right)^{n-1}+x D\left(x^{n-1}\right),
$$

holds for all $x \in \mathcal{R}$. The substitution of $x=e$ in the relation (11) gives $D(e)=0$. Let $y$ be any element of the center $Z(\mathcal{R})$. Putting $x+y$ for $x$ in the above relation, we obtain

$$
\begin{aligned}
2 \sum_{i=0}^{n}\left(\begin{array}{c}
n \\
i
\end{array}\right) D\left(x^{n-i} y^{i}\right)= & \left(\sum_{i=0}^{n-1}\left(\begin{array}{c}
n-1 \\
i
\end{array}\right) D\left(x^{n-1-i} y^{i}\right)\right)\left(x^{*}+y^{*}\right) \\
& +\left(\sum_{i=0}^{n-1}\left(\begin{array}{c}
n-1 \\
i
\end{array}\right) x^{n-1-i} y^{i}\right) D(x+y) \\
& +D(x+y)\left(\sum_{i=0}^{n-1}\left(\begin{array}{c}
n-1 \\
i
\end{array}\right)\left(x^{*}\right)^{n-1-i}\left(y^{*}\right)^{i}\right) \\
& +(x+y)\left(\sum_{i=0}^{n-1}\left(\begin{array}{c}
n-1 \\
i
\end{array}\right) D\left(x^{n-1-i} y^{i}\right)\right) .
\end{aligned}
$$

Using (11) in the above relation and rearranging it in sense of collecting together terms involving equal 
number of factors of $y$, we obtain

$$
\begin{aligned}
0= & 2\left(\begin{array}{c}
n \\
1
\end{array}\right) D\left(x^{n-1} y\right)-\left(\begin{array}{c}
n-1 \\
0
\end{array}\right)\left\{D\left(x^{n-1} y^{*}\right)+x^{n-1} D(y)+D(y)\left(x^{*}\right)^{n-1}+y D\left(x^{n-1}\right)\right\} \\
& -\left(\begin{array}{c}
n-1 \\
1
\end{array}\right)\left\{D\left(x^{n-2} y\right) x^{*}+x^{n-2} y D(x)+D(x)\left(x^{*}\right)^{n-2} y^{*}+x D\left(x^{n-2} y\right)\right\} \\
& +2\left(\begin{array}{c}
n \\
2
\end{array}\right) D\left(x^{n-2} y^{2}\right)-\left(\begin{array}{c}
n-1 \\
1
\end{array}\right)\left\{D\left(x^{n-2} y\right) y^{*}+x^{n-2} y D(y)+D(y)\left(x^{*}\right)^{n-2} y^{*}\right. \\
& \left.+y D\left(x^{n-2} y\right)\right\}-\left(\begin{array}{c}
n-1 \\
2
\end{array}\right)\left\{D\left(x^{n-3} y^{2}\right) x^{*}+x^{n-3} y^{2} D(x)+D(x)\left(x^{*}\right)^{n-3}\left(y^{*}\right)^{2}\right. \\
& \left.+x D\left(x^{n-3} y^{2}\right)\right\}+\cdots+2\left(\begin{array}{c}
n \\
n-1
\end{array}\right) D\left(x y^{n-1}\right)-\left(\begin{array}{c}
n-1 \\
n-2
\end{array}\right)\left\{D\left(x y^{n-2}\right) y^{*}\right. \\
& \left.+x y^{n-2} D(y)+D(y) x^{*}\left(y^{*}\right)^{n-2}+y D\left(x y^{n-2}\right)\right\} \\
& -\left(\begin{array}{c}
n-1 \\
n-1
\end{array}\right)\left\{D\left(y^{n-1}\right) x^{*}+y^{n-1} D(x)+D(x)\left(y^{*}\right)^{n-1}+x D\left(y^{n-1}\right)\right\} .
\end{aligned}
$$

This can be written as

$$
f_{0}(x, y)+f_{1}(x, y)+f_{2}(x, y)+\cdots+f_{n-1}(x, y)=0,
$$

where $f_{i}(x, y)$ stands for the expression of terms involving $i$ factors of $y$. Replace $x$ by $x+2 y, x+3 y$, $\ldots, x+(n-1) y$ in the relation (11) and expressing the resulting system of $n-2$ homogeneous equations of variables $f_{i}(x, y)$ for $i=1,2, \ldots n-1$ together with $(12)$, we see that the coefficient matrix of the system of $n-1$ homogenous equations is a Van-der Monde matrix

$$
\left(\begin{array}{cccc}
1 & 1 & \ldots & 1 \\
2 & 2^{2} & \ldots & 2^{n-1} \\
\vdots & \vdots & \ddots & \vdots \\
n-1 & (n-1)^{2} & \ldots & (n-1)^{n-1}
\end{array}\right)
$$

Since the determinant of this matrix is different from zero, it follows that the system has only a trivial solution, i.e., $f_{i}(x, y)=0$ for $i=1,2, \ldots n-1$. In particular, if $y$ is replaced with the identity element $e$ in $f_{n-2}(x, y)$, we obtain

$$
\begin{aligned}
f_{n-2}(x, e)= & 2\left(\begin{array}{c}
n \\
n-2
\end{array}\right) D\left(x^{2}\right)-\left(\begin{array}{l}
n-1 \\
n-2
\end{array}\right) D(x) x^{*}-\left(\begin{array}{c}
n-1 \\
n-3
\end{array}\right) D\left(x^{2}\right) \\
& -\left(\begin{array}{c}
n-1 \\
n-2
\end{array}\right) x D(x)-\left(\begin{array}{l}
n-1 \\
n-3
\end{array}\right) x^{2} D(e)-\left(\begin{array}{c}
n-1 \\
n-2
\end{array}\right) D(x) x^{*} \\
& -\left(\begin{array}{c}
n-1 \\
n-3
\end{array}\right) D(e)\left(x^{*}\right)^{2}-\left(\begin{array}{l}
n-1 \\
n-3
\end{array}\right) D\left(x^{2}\right)-\left(\begin{array}{l}
n-1 \\
n-2
\end{array}\right) x D(x) .
\end{aligned}
$$

After few calculations and considering the relation $D(e)=0$, we obtain

$$
(n(n-1)-(n-1)(n-2)) D\left(x^{2}\right)=2(n-1)\left(D(x) x^{*}+x D(x)\right) .
$$


Since $\mathcal{R}$ is $2(n-1)$ !-torsion free, it follows from the above relation that

$$
D\left(x^{2}\right)=D(x) x^{*}+x D(x) \text { for all } x \in \mathcal{R} .
$$

Hence $D$ is a Jordan $*$-derivation, which completes the proof.

\section{Results on standard operator algebra $\mathcal{A}(\mathcal{H})$}

Let $\mathcal{H}$ be a real or complex Hilbert space, $\operatorname{dim}(\mathcal{H})>1$. By $\mathcal{B}(\mathcal{H})$ we mean the algebra of all bounded linear operators on $\mathcal{H}$. Denote by $\mathcal{F}(\mathcal{H})$ the subalgebra of bounded finite rank operators. It is to be noted that $\mathcal{F}(\mathcal{H})$ forms a $*$-closed ideal in $\mathcal{B}(\mathcal{H})$. An algebra $\mathcal{A}(\mathcal{H}) \subset \mathcal{B}(\mathcal{H})$ is said to be standard operator algebra in case $\mathcal{F}(\mathcal{H}) \subset \mathcal{A}(\mathcal{H})$. Let us point out that any standard operator algebra is prime, which is a consequence of Hahn-Banach theorem.

The main result of the paper is related to the result below first proved by Šemrl [17] (see also [8]).

Theorem 3.1 [17, Theorem] Let $\mathcal{H}$ be a real or complex Hilbert space, with $\operatorname{dim}(\mathcal{H})>1$, and let $\mathcal{A}(\mathcal{H})$ be a standard operator algebra on $\mathcal{H}$. Suppose that $D: \mathcal{A}(\mathcal{H}) \rightarrow \mathcal{B}(\mathcal{H})$ is a linear Jordan *-derivation. Then there exists a unique linear operator $B \in \mathcal{B}(\mathcal{H})$ such that $D(A)=A B-B A^{*}$ for all $A \in \mathcal{A}(\mathcal{H})$.

TheOREM 3.2 Let $\mathcal{H}$ be a real or complex Hilbert space, with $\operatorname{dim}(\mathcal{H})>1$, and let $\mathcal{A}(\mathcal{H})$ be a standard operator algebra on $\mathcal{H}$. Suppose there exists a linear mapping $D: \mathcal{A}(\mathcal{H}) \rightarrow \mathcal{B}(\mathcal{H})$ such that either

$$
D\left(A^{2 n+1}\right)=D\left(A^{2 n}\right) A^{*}+A^{2 n} D(A), \text { for all } A \in \mathcal{A}(\mathcal{H}),
$$

or

$$
D\left(A^{2 n+1}\right)=D(A)\left(A^{*}\right)^{2 n}+A D\left(A^{2 n}\right), \text { for all } A \in \mathcal{A}(\mathcal{H}) .
$$

In this case $D$ is of the form $D(A)=A B-B A^{*}$ for all $A \in \mathcal{A}(\mathcal{H})$ and some fixed $B \in \mathcal{B}(\mathcal{H})$, which means that $D$ is a Jordan $*$-derivation.

Proof. We have the relation

$$
D\left(A^{2 n+1}\right)=D\left(A^{2 n}\right) A^{*}+A^{2 n} D(A)
$$

for all $A \in \mathcal{A}(\mathcal{H})$. Let us first consider the restriction of $D$ on $\mathcal{F}(\mathcal{H})$. Let $A$ be from $\mathcal{F}(\mathcal{H})$ and let $P \in \mathcal{F}(\mathcal{H})$ be an idempotent operator with $A P=P A=A$. Putting $P$ for $A$ in the relation (13), we obtain

$$
D(P)=D(P) P^{*}+P D(P)
$$

Putting $A+P$ for $A$ in the relation (13), we obtain

$$
\begin{aligned}
\sum_{i=0}^{2 n+1}\left(\begin{array}{c}
2 n+1 \\
i
\end{array}\right) D\left(A^{2 n+1-i} P^{i}\right)= & \left(\sum_{i=0}^{2 n}\left(\begin{array}{c}
2 n \\
i
\end{array}\right) D\left(A^{2 n-i} P^{i}\right)\right)\left(A^{*}+P^{*}\right) \\
& +\left(\sum_{i=0}^{2 n}\left(\begin{array}{c}
2 n \\
i
\end{array}\right) A^{2 n-i} P^{i}\right) D(A+P) .
\end{aligned}
$$


Rearranging the above relation in the sense of collecting together terms involving equal number of factors of $P$, we obtain $\sum_{i=1}^{2 n} f_{i}(A, P)=0$, where

$$
\begin{aligned}
f_{i}(A, P)= & \left(\begin{array}{c}
2 n+1 \\
i
\end{array}\right) D\left(A^{2 n+1-i} P^{i}\right) \\
& -\left(\begin{array}{c}
2 n \\
i
\end{array}\right)\left(D\left(A^{2 n-i} P^{i}\right) A^{*}+A^{2 n-i} P^{i} D(A)\right) \\
& -\left(\begin{array}{c}
2 n \\
i-1
\end{array}\right)\left(D\left(A^{2 n+1-i} P^{i-1}\right) P^{*}+\left(A^{2 n+1-i} P^{i-1}\right) D(P)\right) ;
\end{aligned}
$$

Replacing $A$ by $A+2 P, A+3 P, \ldots, A+2 n P$ in the relation (13) and expressing the resulting system of $2 n$ homogeneous equations of variables $f_{i}(A, P)$ for $i=1,2, \ldots 2 n$, we see that the coefficient matrix of the system of $2 n$ homogenous equations is a Van-der Monde matrix

$$
\left(\begin{array}{cccc}
1 & 1 & \ldots & 1 \\
2 & 2^{2} & \ldots & 2^{2 n} \\
\vdots & \vdots & \ddots & \vdots \\
2 n & (2 n)^{2} & \ldots & (2 n)^{2 n}
\end{array}\right)
$$

Since the determinant of this matrix is different from zero, it follows immediately that the system has only a trivial solution. In particular

$$
\begin{aligned}
f_{2 n-1}(A, P)= & \left(\begin{array}{c}
2 n+1 \\
2 n-1
\end{array}\right) D\left(A^{2}\right)-\left(\begin{array}{c}
2 n \\
2 n-1
\end{array}\right)\left(D(A) A^{*}+A D(A)\right) \\
& +\left(\begin{array}{c}
2 n \\
2 n-2
\end{array}\right)\left(D\left(A^{2}\right) P^{*}+A^{2} D(P)\right) \\
= & 0,
\end{aligned}
$$

and

$$
\begin{aligned}
f_{2 n}(A, P)= & \left(\begin{array}{c}
2 n+1 \\
2 n
\end{array}\right) D(A)-\left(\begin{array}{c}
2 n \\
2 n
\end{array}\right)\left(D(P) A^{*}+P D(A)\right) \\
& +\left(\begin{array}{c}
2 n \\
2 n-1
\end{array}\right)\left(D(A) P^{*}+A D(P)\right) \\
= & 0,
\end{aligned}
$$

The above relations reduces to

$$
\begin{aligned}
& n(2 n+1) D\left(A^{2}\right)= 2 n D(A) A^{*}+2 n A D(A)+n(2 n-1) D\left(A^{2}\right) P^{*} \\
&+n(2 n-1) A^{2} D(P), \\
&(2 n+1) D(A)=D(P) A^{*}+P D(A)+(2 n) D(A) P^{*}+(2 n) A D(P) .
\end{aligned}
$$

Multiplying (15) by $P^{*}$ and using (14), we have

$$
D\left(A^{2}\right) P^{*}=D(A) A^{*}+A D(A) P^{*}
$$


Applying (17) in the relation (15), we obtain

$$
\begin{aligned}
n(2 n+1) D\left(A^{2}\right)= & n(2 n+1) D(A) A^{*}+2 n A D(A) \\
& +n(2 n-1)\left(A D(A) P^{*}+A^{2} D(P)\right)
\end{aligned}
$$

Left multiplication by $A$ in $(16)$ gives

$$
A D(A)=A D(A) P^{*}+A^{2} D(P) .
$$

Applying the above relation in (18), we get

$$
D\left(A^{2}\right)=D(A) A^{*}+A D(A) .
$$

From the relation $(16)$ one can conclude that $D$ maps $\mathcal{F}(\mathcal{H})$ into itself. We have therefore a linear mapping which maps $\mathcal{F}(\mathcal{H})$ into itself satisfying the relation 190 for all $A \in \mathcal{F}(\mathcal{H})$. Hence $D$ is a Jordan $*$-derivation on $\mathcal{F}(\mathcal{H})$. Applying Theorem 3.1 one can conclude that $D$ is of the form

$$
D(A)=A B-B A^{*}
$$

for all $A \in \mathcal{F}(\mathcal{H})$ and some fixed $B \in \mathcal{B}(\mathcal{H})$. It remains to prove that the relation $(20)$ holds for all $A \in \mathcal{A}(\mathcal{H})$ as well. For this purpose we define $D_{0}: \mathcal{A}(\mathcal{H}) \rightarrow \mathcal{B}(\mathcal{H})$ by $D_{0}(A)=A B-B A^{*}$ and consider $D_{1}=D-D_{0}$. Indeed, the mapping $D_{1}$ is linear, satisfies the relation $(13)$ and it vanishes on $\mathcal{F}(\mathcal{H})$. Now we will prove that $D_{1}$ vanishes on $\mathcal{A}(\mathcal{H})$ also. Let $A \in \mathcal{A}(\mathcal{H})$ and $P$ be an idempotent operator of rank one. Let us introduce $S \in \mathcal{A}(\mathcal{H})$ by $S=A+P A P-(A P+P A)$. We have $S P=P S=0$. It is easy to prove that $D_{1}(S)=D_{1}(A)$ and $D_{1}\left(S^{2 n}\right)=D_{1}\left(A^{2 n}\right)$. The relation 13 leads us to

$$
\begin{aligned}
D_{1}\left(S^{2 n}\right) S^{*}+S^{2 n} D_{1}(S) & =D_{1}\left(S^{2 n+1}\right)=D_{1}\left(S^{2 n+1}+P\right)=D_{1}\left((S+P)^{2 n+1}\right) \\
& =D_{1}\left(S^{2 n}\right)(S+P)^{*}+(S+P)^{2 n} D_{1}(S+P) \\
& =D_{1}\left(S^{2 n}\right) S^{*}+D_{1}\left(S^{2 n}\right) P^{*}+\left(S^{2 n}+P\right) D_{1}(S) \\
& =D_{1}\left(S^{2 n}\right) S^{*}+D_{1}\left(S^{2 n}\right) P^{*}+S^{2 n} D_{1}(S)+P D_{1}(S) .
\end{aligned}
$$

Therefore,

$$
D_{1}\left(S^{2 n}\right) P^{*}+P D_{1}(S)=0 ;
$$

Since $D_{1}(S)=D_{1}(A)$ and $D_{1}\left(S^{2 n}\right)=D_{1}\left(A^{2 n}\right)$, above relation can be written as

$$
D_{1}\left(A^{2 n}\right) P^{*}+P D_{1}(A)=0
$$

Replace $A$ by $-A$ in the above relation and compare the relation so obtained with the above relation, we obtain

$$
P D_{1}(A)=0
$$

for all $A \in \mathcal{A}(\mathcal{H})$. Since $P$ is an arbitrary idempotent operator of rank one, we have $D_{1}(A)=0$ for all $A \in \mathcal{A}(\mathcal{H})$, which completes the proof of our theorem. 
Theorem 3.3 Let $\mathcal{H}$ be a real or complex Hilbert space, with $\operatorname{dim}(\mathcal{H})>1$, and let $\mathcal{A}(\mathcal{H})$ be a standard operator algebra on $\mathcal{H}$. Suppose there exists a linear mapping $D: \mathcal{A}(\mathcal{H}) \rightarrow \mathcal{B}(\mathcal{H})$ such that either

$$
D\left(A^{2 n+1}\right)=D\left(A^{2 n}\right) A^{*}-A^{2 n} D(A), \text { for all } A \in \mathcal{A}(\mathcal{H}),
$$

or

$$
D\left(A^{2 n+1}\right)=D(A)\left(A^{*}\right)^{2 n}-A D\left(A^{2 n}\right), \text { for all } A \in \mathcal{A}(\mathcal{H})
$$

and some integer $n \geq 1$. Then $D(A)=0$ for all $A \in \mathcal{A}(\mathcal{H})$.

Proof. We have the relation

$$
D\left(A^{2 n+1}\right)=D\left(A^{2 n}\right) A^{*}-A^{2 n} D(A)
$$

for all $A \in \mathcal{A}(\mathcal{H})$. Let us first consider the restriction of $D$ on $\mathcal{F}(\mathcal{H})$. Let $A$ be from $\mathcal{F}(\mathcal{H})$ and let $P \in \mathcal{F}(\mathcal{H})$ be an idempotent operator with $A P=P A=A$. Putting $P$ for $A$ in the relation (21), we obtain

$$
D(P)=D(P) P^{*}-P D(P)
$$

A right multiplication by $P^{*}$ in the above relation gives

$$
P D(P) P^{*}=0
$$

Left multiplication by $P$ in $(22)$ and combining with the above relation yields

$$
P D(P)=0
$$

Putting $A+P$ for $A$ in the relation (21), we obtain

$$
\begin{aligned}
\sum_{i=0}^{2 n+1}\left(\begin{array}{c}
2 n+1 \\
i
\end{array}\right) D\left(A^{2 n+1-i} P^{i}\right)= & \left(\sum_{i=0}^{2 n}\left(\begin{array}{c}
2 n \\
i
\end{array}\right) D\left(A^{2 n-i} P^{i}\right)\right)\left(A^{*}+P^{*}\right) \\
& -\left(\sum_{i=0}^{2 n}\left(\begin{array}{c}
2 n \\
i
\end{array}\right) A^{2 n-i} P^{i}\right) D(A+P) .
\end{aligned}
$$

Rearranging the above relation in the sense of collecting together terms involving equal number of factors of $P$, we obtain $\sum_{i=1}^{2 n} f_{i}(A, P)=0$, where

$$
\begin{aligned}
f_{i}(A, P)= & \left(\begin{array}{c}
2 n+1 \\
i
\end{array}\right) D\left(A^{2 n+1-i} P^{i}\right) \\
& -\left(\begin{array}{c}
2 n \\
i
\end{array}\right)\left(D\left(A^{2 n-i} P^{i}\right) A^{*}-A^{2 n-i} P^{i} D(A)\right) \\
& -\left(\begin{array}{c}
2 n \\
i-1
\end{array}\right)\left(D\left(A^{2 n+1-i} P^{i-1}\right) P^{*}-\left(A^{2 n+1-i} P^{i-1}\right) D(P)\right)
\end{aligned}
$$


Replace $A$ by $A+P, A+2 P, A+3 P, \ldots, A+2 n P$ in the relation (21) and express the resulting system of $2 n$ homogeneous equations of variables $f_{i}(A, P)$ for $i=1,2, \ldots 2 n$, we see that the coefficient matrix of the system of $2 n$ homogenous equations is a Van-der Monde matrix

$$
\left(\begin{array}{cccc}
1 & 1 & \ldots & 1 \\
2 & 2^{2} & \ldots & 2^{2 n} \\
\vdots & \vdots & \ddots & \vdots \\
2 n & (2 n)^{2} & \ldots & (2 n)^{2 n}
\end{array}\right)
$$

Since the determinant of this matrix is different from zero, it follows immediately that the system has only a trivial solution. In particular

$$
\begin{aligned}
f_{2 n}(A, P)= & \left(\begin{array}{c}
2 n+1 \\
2 n
\end{array}\right) D(A)-\left(\begin{array}{l}
2 n \\
2 n
\end{array}\right)\left(D(P) A^{*}-P D(A)\right) \\
& -\left(\begin{array}{c}
2 n \\
2 n-1
\end{array}\right)\left(D(A) P^{*}-A D(P)\right) \\
= & 0,
\end{aligned}
$$

The above relation reduces to

$$
(2 n+1) D(A)=D(P) A^{*}-P D(A)+(2 n) D(A) P^{*}-(2 n) A D(P) .
$$

Using 233 in the above relation, we get

$$
(2 n+1) D(A)=D(P) A^{*}-P D(A)+(2 n) D(A) P^{*} .
$$

Left multiplication by $P$ and right multiplication by $P^{*}$ in the above relation leads to

$$
P D(A) P^{*}=0 .
$$

Left multiplication by $P$ in the relation (24) and combining (23) together with the above relation, gives

$$
P D(A)=0
$$

Left multiplication by $A$ in the above relation yields

$$
A D(A)=0
$$

Using (25) in 24, we get

$$
(2 n+1) D(A)=D(P) A^{*}+(2 n) D(A) P^{*} .
$$

A right multiplication by $P^{*}$ in the above relation yields $D(A) P^{*}=D(P) A^{*}$, and hence combining the latter relation with (27), we obtain

$$
D(A)=D(A) P^{*}
$$


From the relation $(28)$ one can conclude that $D$ maps $\mathcal{F}(\mathcal{H})$ into itself. We have therefore a linear mapping which maps $\mathcal{F}(\mathcal{H})$ into itself satisfying the relation 26 for all $A \in \mathcal{F}(\mathcal{H})$. Applying Lemma 2.1 one can conclude that $D(A)=0$ for all $A \in \mathcal{F}(\mathcal{H})$.

It remains to prove that $D(A)=0$ holds for all $A \in \mathcal{A}(\mathcal{H})$ as well. Indeed, the mapping $D$ on $\mathcal{A}(\mathcal{H})$ is linear and satisfies the relation 21). Our aim is to prove that $D$ vanishes on $\mathcal{A}(\mathcal{H})$ also. Let $A \in \mathcal{A}(\mathcal{H})$ and $P$ be an idempotent operator of rank one. Let us introduce $S \in \mathcal{A}(\mathcal{H})$ by $S=A+P A P-(A P+P A)$. We have $S P=P S=0$. It is easy to prove that $D(S)=D(A)$ and $D\left(S^{2 n}\right)=D\left(A^{2 n}\right)$. The relation 21 leads us to

$$
\begin{aligned}
D\left(S^{2 n}\right) S^{*}-S^{2 n} D(S) & =D\left(S^{2 n+1}\right)=D\left(S^{2 n+1}+P\right)=D\left((S+P)^{2 n+1}\right) \\
& =D\left(S^{2 n}\right)(S+P)^{*}-(S+P)^{2 n} D(S+P) \\
& =D\left(S^{2 n}\right) S^{*}+D\left(S^{2 n}\right) P^{*}-\left(S^{2 n}+P\right) D(S) \\
& =D\left(S^{2 n}\right) S^{*}+D\left(S^{2 n}\right) P^{*}-S^{2 n} D(S)-P D(S) .
\end{aligned}
$$

Therefore,

$$
D\left(S^{2 n}\right) P^{*}-P D(S)=0
$$

Since $D(S)=D(A)$ and $D\left(S^{2 n}\right)=D\left(A^{2 n}\right)$, the above relation can be written as

$$
D\left(A^{2 n}\right) P^{*}-P D(A)=0
$$

Replace $A$ by $-A$ in the above relation and compare the relation so obtained with the above relation, we obtain

$$
P D(A)=0
$$

for all $A \in \mathcal{A}(\mathcal{H})$. Since $P$ is an arbitrary idempotent operator of rank one, we have $D(A)=0$ for all $A \in \mathcal{A}(\mathcal{H})$, which completes the proof of the theorem.

Theorem 3.4 Let $\mathcal{H}$ be a real or complex Hilbert space, with $\operatorname{dim}(\mathcal{H})>1$, and let $\mathcal{A}(\mathcal{H})$ be a standard operator algebra on $\mathcal{H}$. Suppose there exist a linear mappings $D, G: \mathcal{A}(\mathcal{H}) \rightarrow \mathcal{B}(\mathcal{H})$ such that either the relations

$$
\begin{aligned}
& D\left(A^{2 n+1}\right)=D\left(A^{2 n}\right) A^{*}+A^{2 n} G(A), \\
& G\left(A^{2 n+1}\right)=G\left(A^{2 n}\right) A^{*}+A^{2 n} D(A)
\end{aligned}
$$

hold for all $A \in \mathcal{A}(\mathcal{H})$, or the relations

$$
\begin{aligned}
& D\left(A^{2 n+1}\right)=D(A)\left(A^{*}\right)^{2 n}+A G\left(A^{2 n}\right), \\
& G\left(A^{2 n+1}\right)=G(A)\left(A^{*}\right)^{2 n}+A D\left(A^{2 n}\right)
\end{aligned}
$$

hold for all $A \in \mathcal{A}(\mathcal{H})$. In both the cases $D(A)=G(A)=A B-B A^{*}$ for all $A \in \mathcal{A}(\mathcal{H})$ and some fixed $B \in \mathcal{B}(\mathcal{H})$, which means that $D$ and $G$ are Jordan $*$-derivations. 
Proof. We will restrict our attention on the first system of relations, the proof in case when $\mathcal{A}(\mathcal{H})$ satisfies the second system of relations is similar and will therefore be omitted. We have

$$
\begin{aligned}
& D\left(A^{2 n+1}\right)=D\left(A^{2 n}\right) A^{*}+A^{2 n} G(A), \\
& G\left(A^{2 n+1}\right)=G\left(A^{2 n}\right) A^{*}+A^{2 n} D(A)
\end{aligned}
$$

hold for all $A \in \mathcal{A}(\mathcal{H})$. Subtracting the above relations, we get

$$
T\left(A^{2 n+1}\right)=T\left(A^{2 n}\right) A^{*}-A^{2 n} T(A),
$$

where $T=D-G$. Using Theorem 3.3 , we conclude that $T(A)=0$ for all $A \in \mathcal{A}(\mathcal{H})$, which implies $D=G$. This assertion enables us to combine the given two relations into only one relation

$$
D\left(A^{2 n+1}\right)=D\left(A^{2 n}\right) A^{*}+A^{2 n} D(A)
$$

for all $A \in \mathcal{A}(\mathcal{H})$. From Theorem 3.2 it follows that $D(A)=G(A)=A B-B A^{*}$ for all $A \in \mathcal{A}(\mathcal{H})$, and hence the proof is complete.

Our next result is in the spirit of the conjecture 2.5.

Theorem 3.5 Let $\mathcal{H}$ be a real or complex Hilbert space, with $\operatorname{dim}(\mathcal{H})>1$, and let $\mathcal{A}(\mathcal{H})$ be a standard operator algebra on $\mathcal{H}$. Suppose there exists a linear mapping $D: \mathcal{A}(\mathcal{H}) \rightarrow \mathcal{B}(\mathcal{H})$ such that

$$
2 D\left(A^{n}\right)=D\left(A^{n-1}\right) A^{*}+A^{n-1} D(A)+D(A)\left(A^{*}\right)^{n-1}+A D\left(A^{n-1}\right)
$$

for all $A \in \mathcal{A}(\mathcal{H})$. In this case $D$ is of the form $D(A)=A B-B A^{*}$ for all $A \in \mathcal{A}(\mathcal{H})$ and some fixed $B \in \mathcal{B}(\mathcal{H})$, which means that $D$ is a Jordan $*$-derivation.

Proof. Let us first consider the restriction of $D$ on $\mathcal{F}(\mathcal{H})$. Let $A$ be from $\mathcal{F}(\mathcal{H})$ and let $P \in \mathcal{F}(\mathcal{H})$ be an idempotent operator with $A P=P A=A$. Putting $P$ for $A$ in the relation $[30$, we obtain

$$
D(P)=D(P) P^{*}+P D(P)
$$

Putting $A+P$ for $A$ in the relation $(30)$, we obtain

$$
\begin{aligned}
2 \sum_{i=0}^{n}\left(\begin{array}{c}
n \\
i
\end{array}\right) D\left(A^{n-i} P^{i}\right)= & \left(\sum_{i=0}^{n-1}\left(\begin{array}{c}
n-1 \\
i
\end{array}\right) D\left(A^{n-1-i} P^{i}\right)\right)\left(A^{*}+P^{*}\right) \\
& +\left(\sum_{i=0}^{n-1}\left(\begin{array}{c}
n-1 \\
i
\end{array}\right) A^{n-1-i} P^{i}\right) D(A+P) \\
& +D(A+P)\left(\sum_{i=0}^{n-1}\left(\begin{array}{c}
n-1 \\
i
\end{array}\right)\left(A^{*}\right)^{n-1-i}\left(P^{*}\right)^{i}\right) \\
& +(A+P)\left(\sum_{i=0}^{n-1}\left(\begin{array}{c}
n-1 \\
i
\end{array}\right) D\left(A^{n-1-i} P^{i}\right)\right) .
\end{aligned}
$$


Rearranging the above relation in the sense of collecting together terms involving equal number of factors of $P$, we obtain $\sum_{i=1}^{n-1} f_{i}(A, P)=0$, where

$$
\begin{aligned}
f_{i}(A, P)= & 2\left(\begin{array}{c}
n \\
i
\end{array}\right) D\left(A^{n-i} P^{i}\right)-\left(\begin{array}{c}
n-1 \\
i
\end{array}\right)\left(D\left(A^{n-1-i} P^{i}\right) A^{*}+A^{n-1-i} P^{i} D(A)\right) \\
& -\left(\begin{array}{c}
n-1 \\
i-1
\end{array}\right)\left(D\left(A^{n-i} P^{i-1}\right) P^{*}+\left(A^{n-i} P^{i-1}\right) D(P)\right) \\
& -\left(\begin{array}{c}
n-1 \\
i
\end{array}\right)\left(D(A)\left(A^{*}\right)^{n-1-i}\left(P^{*}\right)^{i}+A D\left(A^{n-1-i} P^{i}\right)\right) \\
& -\left(\begin{array}{c}
n-1 \\
i-1
\end{array}\right)\left(D(P)\left(A^{*}\right)^{n-i}\left(P^{*}\right)^{i-1}+P D\left(A^{n-i} P^{i-1}\right)\right)
\end{aligned}
$$

Replacing $A$ by $A+2 P, A+3 P, \ldots, A+(n-1) P$ in the relation $(30)$ and expressing the resulting system of $n-1$ homogeneous equations of variables $f_{i}(A, P)$ for $i=1,2, \ldots n-1$, we see that the coefficient matrix of the system of $n-1$ homogenous equations is a Van-der Monde matrix

$$
\left(\begin{array}{cccc}
1 & 1 & \ldots & 1 \\
2 & 2^{2} & \ldots & 2^{n-1} \\
\vdots & \vdots & \ddots & \vdots \\
n-1 & (n-1)^{2} & \ldots & (n-1)^{n-1}
\end{array}\right)
$$

Since the determinant of this matrix is different from zero, it follows immediately that the system has only a trivial solution. In particular

$$
\begin{aligned}
f_{n-1}(A, P)= & 2\left(\begin{array}{c}
n \\
n-1
\end{array}\right) D(A)-\left(\begin{array}{l}
n-1 \\
n-1
\end{array}\right) D(P) A^{*}-\left(\begin{array}{l}
n-1 \\
n-2
\end{array}\right) D(A) P^{*} \\
& -\left(\begin{array}{c}
n-1 \\
n-1
\end{array}\right) P D(A)-\left(\begin{array}{l}
n-1 \\
n-2
\end{array}\right) A D(P)-\left(\begin{array}{l}
n-1 \\
n-1
\end{array}\right) D(A) P^{*} \\
& -\left(\begin{array}{l}
n-1 \\
n-2
\end{array}\right) D(P) A^{*}-\left(\begin{array}{l}
n-1 \\
n-1
\end{array}\right) A D(P)-\left(\begin{array}{l}
n-1 \\
n-2
\end{array}\right) P D(A) .
\end{aligned}
$$

The above relation reduces to

$$
2 D(A)=D(A) P^{*}+A D(P)+D(P) A^{*}+P D(A)
$$

Replace $A$ by $A^{2}$ in the above relation, to obtain

$$
2 D\left(A^{2}\right)=D\left(A^{2}\right) P^{*}+A^{2} D(P)+D(P)\left(A^{*}\right)^{2}+P D\left(A^{2}\right)
$$

As the previously mentioned system of $n-1$ homogeneous equtions has only a trivial solution, we also obtain

$$
\begin{aligned}
f_{n-2}(A, P)= & 2\left(\begin{array}{c}
n \\
n-2
\end{array}\right) D\left(A^{2}\right)-\left(\begin{array}{l}
n-1 \\
n-2
\end{array}\right) D(A) A^{*}-\left(\begin{array}{l}
n-1 \\
n-3
\end{array}\right) D\left(A^{2}\right) P^{*} \\
& -\left(\begin{array}{l}
n-1 \\
n-2
\end{array}\right) A D(A)-\left(\begin{array}{l}
n-1 \\
n-3
\end{array}\right) A^{2} D(P)-\left(\begin{array}{l}
n-1 \\
n-2
\end{array}\right) D(A) A^{*} \\
& -\left(\begin{array}{l}
n-1 \\
n-3
\end{array}\right) D(P)\left(A^{*}\right)^{2}\left(\begin{array}{l}
n-1 \\
n-2
\end{array}\right) A D(A)-\left(\begin{array}{l}
n-1 \\
n-3
\end{array}\right) P D\left(A^{2}\right) \\
= & 0 .
\end{aligned}
$$


The above relation reduces to

$$
\begin{aligned}
n(n-1) D\left(A^{2}\right)= & 2(n-1)\left(D(A) A^{*}+A D(A)\right) \\
& +\left(\begin{array}{c}
n-1 \\
n-3
\end{array}\right)\left(D\left(A^{2}\right) P^{*}+A^{2} D(P)+D(P)\left(A^{*}\right)^{2}+P D\left(A^{2}\right)\right) .
\end{aligned}
$$

Applying the relation 33 in the above relation, we obtain

$$
n(n-1) D\left(A^{2}\right)=2(n-1)\left(D(A) A^{*}+A D(A)\right)+(n-1)(n-2) D\left(A^{2}\right),
$$

which reduces to

$$
D\left(A^{2}\right)=D(A) A^{*}+A D(A)
$$

From the relation $(32)$ one can conclude that $D$ maps $\mathcal{F}(\mathcal{H})$ into itself. We therefore have a linear mapping $D$ which maps $\mathcal{F}(\mathcal{H})$ into itself satisfying the relation $(34)$ for all $A \in \mathcal{F}(\mathcal{H})$. Hence $D$ is a Jordan $*$-derivation on $\mathcal{F}(\mathcal{H})$. Applying Theorem 3.1 one can conclude that $D$ is of the form

$$
D(A)=A B-B A^{*}
$$

for all $A \in \mathcal{F}(\mathcal{H})$ and some fixed $B \in \mathcal{B}(\mathcal{H})$. It remains to prove that the relation (35) holds for all $A \in \mathcal{A}(\mathcal{H})$ as well. For this purpose we define $D_{0}: \mathcal{A}(\mathcal{H}) \rightarrow \mathcal{B}(\mathcal{H})$ by $D_{0}(A)=A B-B A^{*}$ and consider $D_{1}=D-D_{0}$. Indeed, the mapping $D_{1}$ is linear, satisfies the relation $(30)$ and it vanishes on $\mathcal{F}(\mathcal{H})$. Now we prove that $D_{1}$ vanishes on $\mathcal{A}(\mathcal{H})$ also. Let $A \in \mathcal{A}(\mathcal{H})$ and $P$ be an idempotent operator of rank one. Let us introduce $S \in \mathcal{A}(\mathcal{H})$ by $S=A+P A P-(A P+P A)$. We have $S P=P S=0$. It is easy to prove that $D_{1}(S)=D_{1}(A)$ and $D_{1}\left(S^{n-1}\right)=D_{1}\left(A^{n-1}\right)$. By the relation 30 we now have

$$
\begin{aligned}
& D_{1}\left(S^{n-1}\right) S^{*}+S^{n-1} D_{1}(S)+D_{1}(S)\left(S^{*}\right)^{n-1}+S D_{1}\left(S^{n-1}\right) \\
&= 2 D_{1}\left(S^{n}\right)=2 D_{1}\left(S^{n}+P\right)=2 D_{1}\left((S+P)^{n}\right) \\
&= D_{1}\left((S+P)^{n-1}\right)(S+P)^{*}+(S+P)^{n-1} D_{1}(S+P) \\
&+D_{1}(S+P)\left((S+P)^{*}\right)^{n-1}+(S+P) D_{1}\left((S+P)^{n-1}\right) \\
&= D_{1}\left(S^{n-1}\right) S^{*}+D_{1}\left(S^{n-1}\right) P^{*}+S^{n-1} D_{1}(S)+P D_{1}(S) \\
&+D_{1}(S)\left(S^{*}\right)^{n-1}+D_{1}(S) P^{*}+S D_{1}\left(S^{n-1}\right)+P D_{1}\left(S^{n-1}\right) .
\end{aligned}
$$

From the above relation it follows that

$$
D_{1}\left(S^{n-1}\right) P^{*}+P D_{1}(S)+D_{1}(S) P^{*}+P D_{1}\left(S^{n-1}\right)=0 .
$$

Since $D_{1}(S)=D_{1}(A)$, we can rewrite the above relation as

$$
D_{1}\left(A^{n-1}\right) P^{*}+P D_{1}(A)+D_{1}(A) P^{*}+P D_{1}\left(A^{n-1}\right)=0 .
$$

Putting $2 A$ for $A$ in the above relation, we obtain

$$
2^{n-1} D_{1}\left(A^{n-1}\right) P^{*}+2 P D_{1}(A)+2 D_{1}(A) P^{*}+2^{n-1} P D_{1}\left(A^{n-1}\right)=0 .
$$

In case $n=2$, the relation 36 implies that

$$
P D_{1}(A)+D_{1}(A) P^{*}=0 .
$$


In casen $>2$, the relations $(36)$ and $(37)$ give the relation (38). Multiplying the above relation from left side by $P$ and right side by $P^{*}$, we obtain

$$
P D_{1}(A) P^{*}=0 .
$$

Left multiplication by $P$ in the relation $(38)$ and using using the above relation, we obtain

$$
P D_{1}(A)=0 .
$$

for all $A \in \mathcal{A}(\mathcal{H})$. Since $P$ is an arbitrary an idempotent operator of rank one, we have $D_{1}(A)=0$ for all $A \in \mathcal{A}(\mathcal{H})$, which completes the proof of the theorem.

We conclude the paper with the following purely algebraic conjecture.

Conjecture 3.6 Let $\mathcal{R}$ be a semiprime $*$-ring with a suitable torsion restriction and $D, G: \mathcal{R} \rightarrow \mathcal{R}$ be additive mappings such that either the relations

$$
\begin{aligned}
& D\left(x^{2 n+1}\right)=D\left(x^{2 n}\right) x^{*}+x^{2 n} G(x), \\
& G\left(x^{2 n+1}\right)=G\left(x^{2 n}\right) x^{*}+x^{2 n} D(x)
\end{aligned}
$$

hold for all $x \in \mathcal{R}$, or the relations

$$
\begin{aligned}
& D\left(x^{2 n+1}\right)=D(x)\left(x^{*}\right)^{2 n}+x G\left(x^{2 n}\right), \\
& G\left(x^{2 n+1}\right)=G(x)\left(x^{*}\right)^{2 n}+x D\left(x^{2 n}\right)
\end{aligned}
$$

hold for all $x \in \mathcal{R}$ and some fixed integer $n \geq 1$. Then $D$ and $G$ are Jordan $*$-derivations and $D=G$.

Acknowledgement The authors are thankful to the referee for his/her valuable comments.

\section{References}

[1] S. Ali AND A. Fošner, On Jordan $(\alpha, \beta)^{*}$-derivation in semiprime $*$-rings, Int. J. Algebra, 4 (2010) 99-108.

[2] M. Ashraf, N. Rehman and Shakir Ali, On Lie ideals and Jordan generalized derivations of prime rings, Indian J. Pure Appl. Math., 34 (2003) 291-294.

[3] M. Ashraf And N. Rehman, On Jordan ideals and Jordan derivations of prime rings, Demonstratio Math., 37 (2004) 275-284.

[4] M. BREŠAR, Jordan derivations on semiprime rings, Proc. Amer. Math. Soc., 104 (1988) 1003-1006.

[5] M. BREŠAR, Jordan mappings of semiprime rings, J. Algebra, 127 (1989) 218-228.

[6] M. Brešar and J. Vukman, Jordan derivations on prime rings, Bull. Austral. Math. Soc., 37 (1988) 321-322. 
[7] M. BrešAR AND J. Vukman, On some additive mappings in rings with involution, Aequationes Math., 38 (1989) 178-185.

[8] M. BRešAR AND B. ZALAR, On the structure of Jordan *-derivation, Colloq. Math., 63 (1992) 163-171.

[9] J. Cusack, Jordan derivations on rings, Proc. Amer. Math. Soc., 53 (1975) 321-324.

[10] M. FošneR AND D. ILIŠEviČ, On Jordan triple derivations and related mappings, Mediterr. J. Math., 5 (2008) 415-427.

[11] I. N. Herstein, Jordan derivations of prime rings, Proc. Amer. Math. Soc., 8 (1957) 11041119.

[12] I. N. Herstein, Topics in Ring Theory, The University of Chicago Press, Chicago, London, 1969 .

[13] I. N. Herstein, Rings with Involution, The University of Chicago Press, Chicago, London, 1979 .

[14] K. H. Kim And Y. H. LeE, A note on $*$-derivations on $*$-prime rings, Int. Math. Forum, 12 (2017) 391-398.

[15] P. ŠemrL, On Jordan *-derivations and an application, Colloq. Math., 59 (1990) 241-251.

[16] P. ŠEmRL, Quadratic functionals and Jordan *-derivations, Studia Math., 97 (1991) 157-165.

[17] P. ŠEMRL, Jordan *-derivations of standard operator algebras, Proc. Amer. Math. Soc., 120 (1994) 515-518.

[18] N. ŠIROVNIK, On certain functional equation in semiprime rings and standard operator algebras, Cubo, 16 (2014) 73-80.

[19] N. ŠIrovnik And J. Vukman, On certain functional equation in semiprime rings, Algebra Colloq., 23 (2016) 65-70.

[20] N. ŠIRovNIK, On functional equations related to derivations in semiprime rings and standard operator algebras, Glas. Mat. Ser. III, 47 (2012) 95-104.

[21] J. Vukman, Some remarks on derivations in semiprime rings and standard operator algebras, Glas. Mat. Ser. III, 46 (2011) 43-48.

[22] J. Vukman, Identities with derivations and automorphisms on semiprime rings, Int. J. Math. Math. Sci., 7 (2005) 1031-1038.

[23] J. VUKman, Identities related to derivations and centralizers on standard operator algebras, Taiwanese J. Math., 11 (2007) 255-265.

[24] J. Vukman, A note on Jordan *-derivations in semiprime rings with involution, Int. Math. Forum, 13 (2006) 617-622. 